\title{
Caracterización fisicoquímica en poscosecha de diferentes materiales de lima ácida Tahití (Citrus latifolia Tanaka) para exportación
}

\section{Postharvest physicochemical characterization of different materials of Tahiti acid lime (Citrus latifolia Tanaka) for exports}
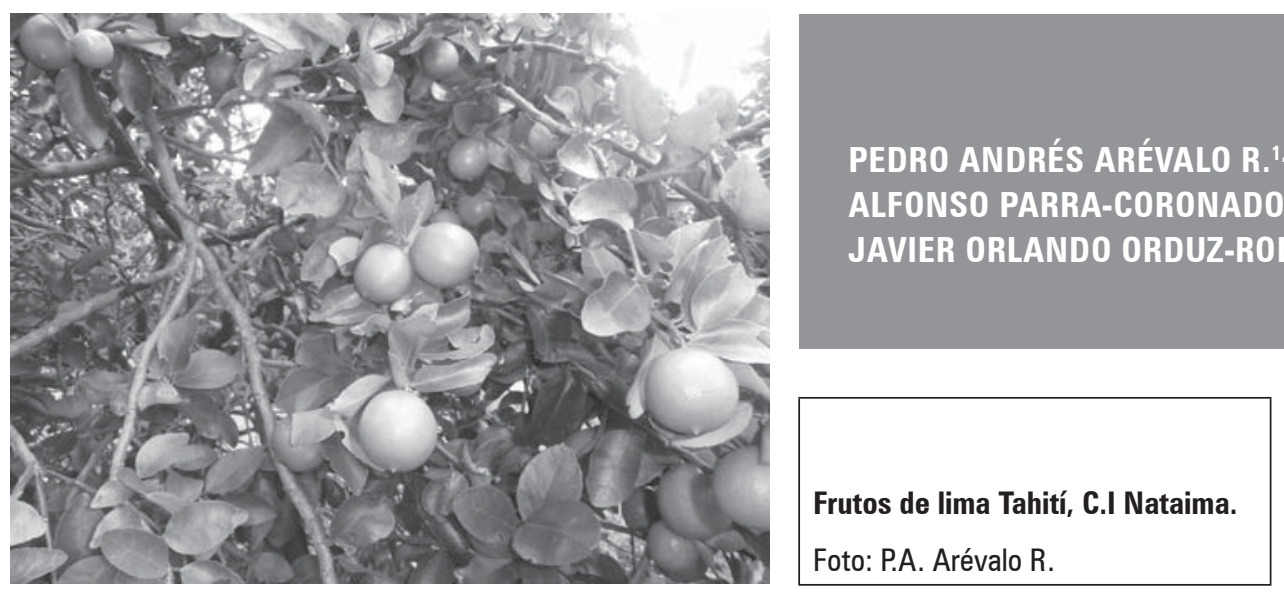

\section{RESUMEN}

Colombia exportó 5.912 t de lima ácida Tahití durante 2015 hacia Estados Unidos y los países del Caribe, donde se redujo la producción por la enfermedad conocida como "verdeamiento" de los cítricos o "Huanglongbing". En el presente estudio se analizaron las características físicas y químicas de frutos provenientes de seis patrones: Sunki $\times$ English, Volkameriano, Kryder, Cleopatra, Citrumelo y Carrizo, almacenados durante 60 días en condiciones ambientales de la ciudad de Bogotá, sin refrigeración. El análisis estadístico se realizó mediante ANOVA seguido por una separación de medias LSD $(\alpha=0,05)$, lo que mostró que las variables cumplen con las exigencias de los mercados de exportación para todos los clones, así: diámetro transversal

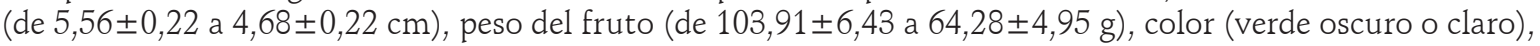
porcentaje del jugo ( $>27 \%$ del peso del fruto), porcentaje de acidez $(<5 \%)$ y firmeza $(<0,22065 \mathrm{kPa})$. Los patrones evaluados cumplen con la norma de exportación y conservan sus características físicas y químicas durante el almacenamiento hasta los 30 días después de cosecha (ddc), destacándose 'Kryder' que las conserva hasta los 45 ddc. Anticipar la cosecha 1, 3, 6 y 9 días antes del momento oportuno de la cosecha comercial, no tiene efectos adicionales sobre la calidad de los frutos y no prolonga el tiempo de almacenamiento.

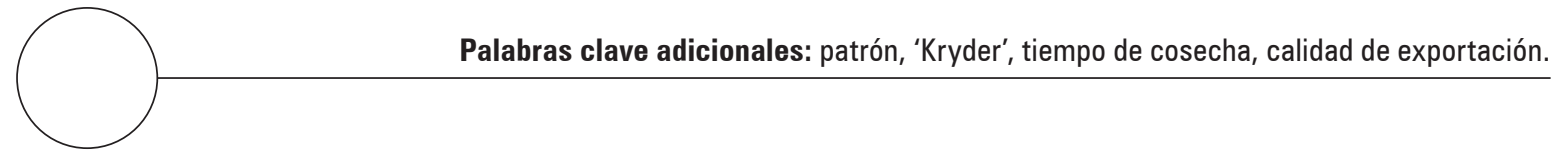

Facultad de Ingeniería, Departamento de Ingeniería Civil y Agrícola, Programa de Maestría en Ingeniería Agrícola, Universidad Nacional de Colombia, Bogotá (Colombia).

2 Facultad de Ingeniería, Departamento de Ingeniería Civil y Agrícola, Universidad Nacional de Colombia, Bogotá (Colombia).

3 Corporación Colombiana de Investigación Agropecuaria (Corpoica), C.I. La Libertad, Villavicencio (Colombia).

4 Autor para correspondencia. paarevalor@unal.edu.co 


\section{ABSTRACT}

Colombia exported 5,912 t of Tahiti lemons to the United States and the Caribbean in 2015, where the production was reduced by the presence of citrus greening or Huanglongbing disease. During this study the physicochemical characteristics of the fruits from six rootstocks: Sunki $\times$ English, Volkameriano, Kryder, Cleopatra, Citrumelo and Carrizo were evaluated at different intervals up to 60 days at the conditions of Bogota, Colombia without refrigeration. Data analysis using ANOVA followed by post-hoc test LSD $(\alpha=0.05)$. The analysis demonstrated that all the rootstocks exceeded the export market expectations for all the clones: equatorial diameter $(4.68 \pm 0.22$ to $5.56 \pm 0.22$ $\mathrm{cm}$ ), fruit weight $(64.28 \pm 4.95$ to $103.91 \pm 6.43 \mathrm{~g}$ ), color (dark green or light green), juice percent ( $>27 \%$ of fruit weight), citric acid percentage $(<5 \%)$ and firmness $(<0.22065 \mathrm{kPa})$. The evaluated rootstocks comply with the export standards and retain their physical and chemical characteristics during the storage until 30 days after harvest (DAH), standing out 'Kryder' that preserves them up to $45 \mathrm{DAH}$. Anticipating harvest 1, 3, 6 and 9 days before the timing of the commercial harvest, has no additional effects on the quality of the fruits and does not prolong the storage time.

Additional key words: rootstock, 'Kryder', harvest time, export quality.

Fecha de recepción: 10-07-2016 Aprobado para publicación: 24-11-2016

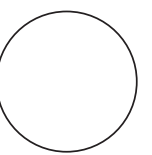

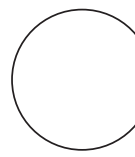

La lima ácida Tahití, originaria de Persia (Irán) en el Sureste Asiático, se cultiva en 21 departamentos de Colombia, donde existen condiciones ambientales favorables para la producción de frutos de calidad: altitud entre 0 y $1.600 \mathrm{msnm}$, temperaturas entre 23 y $34^{\circ} \mathrm{C}$, precipitación entre 900 y $1.200 \mathrm{~mm}$ y luminosidad de $1.900 \mathrm{~h}_{\text {año-1 }}$ (Dorado, 2011).

En Colombia se producen principalmente dos limas ácidas: el limón Pajarito o Mexicano (Citrus aurantifolia) y la lima ácida Tahití (C. latifolia). Los colombianos demandan frutos provenientes del departamento del Tolima por el alto contenido de jugo, como el atributo más apetecido, mientras que para el mercado de exportación el mayor interés es la frescura y la apariencia del fruto, color verde intenso uniforme en toda la corteza, libre de áreas blancuzcas, sin golpes de sombra, porque su principal uso es en la preparación de cocteles (Bassan et al., 2016). Durante 2007 se exportaron 3.596 t y en 2014 aproximadamente 4.529 $t$, alcanzando una cifra significativa en el mercado de frutas de exportación de 27.300 t durante los últimos 13 años, convirtiéndose en una oportunidad de mercado para los agricultores (Hernández et al., 2014).

Por varias razones en la citricultura, sobre todo sanitarias y por disminuir el periodo largo de la juvenilidad, que puede durar hasta más de 10 años, el injerto de la variedad sobre diferentes tipos de patrones es una solución a muchos problemas sanitarios y agronómicos (Orduz-Rodríguez, 2012). En estudios realizados en México para determinar la calidad de la lima en diferentes portainjertos (citrumelo 'Swingle', limón 'Macrophylla', naranjo 'Agrio', citrange 'C35' y 'Troyer', limón 'Volkameriano', lima 'Dulce de Palestina' y naranjo 'Flying Dragon') encontraron que la interacción portainjerto/variedad modificó características físicas del fruto en las variables de masa, diámetro, grosor de la cáscara y firmeza del fruto. Sin embargo se recomendó a los productores sembrar tres o más portainjertos en su huertos, ya que el porcentaje de exportación en todos los estudiados fue superior al 80\% (Berdeja-Arbeu et al., 2016).

En los últimos años la producción de lima en Estados Unidos, Brasil y en las islas del Caribe se ha visto afectada por la enfermedad conocida como el "verdeamiento de los cítricos" o "Huanglongbing" cuyo agente causal es la bacteria Candidatus Liberibacter spp., transmitida por un insecto vector Diaphorina citri (Ebratt et al., 2011a, b; Soares et al., 2015). Como Colombia está libre de la enfermedad, se presenta una gran oportunidad para incrementar sus exportaciones, luego es importante estudiar las características físicas y químicas de los frutos que se producen en las diferentes zonas del país. Por esta razón, el objetivo 
de la presente investigación fue estudiar la influencia de las condiciones de cosecha y tiempo de almacenamiento en las características fisicoquímicas de los frutos de lima ácida Tahití injertada sobre seis diferentes patrones, desarrollados por Corpoica, para que cumplan con los estándares de calidad para exportación.

\section{MATERIALES Y MÉTODOS}

El presente trabajo se realizó con el apoyo de la Corporación Colombiana de Investigación Agropecuaria (Corpoica). La toma de muestras de frutos se realizó en Centro de Investigación Nataima, ubicado en el municipio El Espinal, departamento del Tolima, donde existe un huerto sembrado con seis patrones de lima ácida Tahití, así: S×E, Volkameriano, Kryder, Cleopatra, Citrumelo y Carrizo. Los patrones están plantados en bloques al azar con cuatro replicaciones, de seis árboles por parcela.

El municipio El Espinal, ubicado a $4^{\circ} 09^{\prime} \mathrm{N}$ y $74^{\circ} 09^{\prime}$ W, presenta formación ecológica de bosque seco tropical (Bs-T), con régimen de lluvias bimodal y precipitaciones acumulada de $1.476 \mathrm{~mm}$ anuales (IGAC, 2016). Las temperaturas son óptimas para el desarrollo de la lima Tahití, con mínima de $22^{\circ} \mathrm{C}$ y máxima de $35^{\circ} \mathrm{C}$ (Coelho et al., 2004; Hernández et al., 2014). El municipio El Espinal se encuentra a 335 $\mathrm{msnm}$, temperatura media de $28^{\circ} \mathrm{C}$, humedad relativa media de $72 \%$, brillo solar promedio de $6 \mathrm{~h} \mathrm{dia}^{-1} \mathrm{y}$ velocidad del viento anual de $7,9 \mathrm{~km} \mathrm{~h}^{-1}$ en promedio (Hernández et al., 2014; Dorado et al., 2015).

\section{Diseño experimental y tratamiento de datos}

Se utilizó el mismo diseño experimental que presentaba el huerto de lima ácida Tahití plantado en el Centro Experimental Nataima. Huerto sembrado en bloques al azar, con seis patrones diferentes de lima por parcela, en cuatro replicaciones. La toma de muestras se realizó teniendo en cuenta la época de floración, cosechando a mano los frutos entre los 110 y $120 \mathrm{~d}$ después de floración, con base en los estudios previos realizados por Corpoica (Caicedo, 2000) y por Cañizares et al. (2012). Durante la recolección en campo los frutos fueron cosechados seleccionando aquellos de apariencia redonda, que parezcan pesados para su tamaño, cáscara firme, piel lisa, brillantes y de color verde oscuro intenso, tal como lo recomiendan los manuales de poscosecha (Rodríguez, 2002; Santos et al., 2015).
Se realizaron dos muestreos: el primero, en el momento de la cosecha comercial oportuna (110-120 d después de floración), colectando 360 frutos en total, correspondientes a tres limones por árbol y patrón, para cinco tiempos de almacenamiento, para cada uno de los seis patrones en las cuatro replicaciones. En el segundo, se recolectaron 1.152 frutos en total, cosechando tres frutos por árbol a cada uno de los seis patrones, en cuatro momentos diferentes 1, 3, 6 y 9 días antes de cosecha oportuna (dac), para cuatro lecturas de tiempo de almacenamiento $(0,15,30$ y 45 días después de cosecha, ddc) y cuatro replicaciones.

Por último, los frutos recolectados fueron almacenados en una bodega sin refrigeración y bajo condiciones ambientales de la ciudad de Bogotá (temperatura media de $14^{\circ} \mathrm{C}$ y humedad relativa media de $70 \%$ ).

\section{Variables y medidas}

Las variables estudiadas fueron: peso individual del fruto, tamaño, grosor de la cáscara, color, porcentaje de jugo, contenido de sólidos solubles totales (SST), acidez total titulable (ATT) y firmeza. El peso individual de los frutos (g) se determinó en una balanza

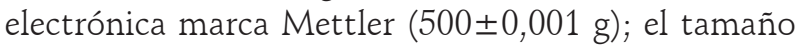
de los frutos se estableció mediante la medición de los diámetros transversal y longitudinal, así como el grosor de la cáscara, con medidor milimétrico de Vernier de mano $(15 \mathrm{~cm}, \pm 0,005 \mathrm{~mm})$. El color de los frutos se determinó con la ayuda de una carta colorimétrica (Fig. 1), con base en la Norma Técnica Colombiana 4087 (Icontec, 1997), que regula la exportación de la lima Tahití.

El jugo se extrajo manualmente con ayuda de un exprimidor de marca Oster de $200 \mathrm{~mL}$ y su peso se relacionó con el peso del fruto, para así determinar el porcentaje de jugo. El contenido de sólidos solubles totales (SST) se determinó directamente mediante un refractómetro marca Atago, modelo RHB-3, Serie 13316 (precisión $\pm 0,2 \%$ ) registrando el porcentaje de sólidos solubles totales ('Brix). La acidez total titulable (ATT), como porcentaje de ácido cítrico, se determinó por titulación de una muestra de jugo con hidróxido de sodio al 0,098 N, utilizando fenolftaleína al 1\% como indicador. La firmeza o resistencia a la penetración de los frutos se determinó con un penetrómetro marca Chatillon serie TCD 2000 (225lbf, 1.000 kN) y precisión 1,5 g. Para facilitar la medición de la firmeza de las limas se estableció la tabla 1, de acuerdo con la norma NTC 4087 (Icontec, 1997). 


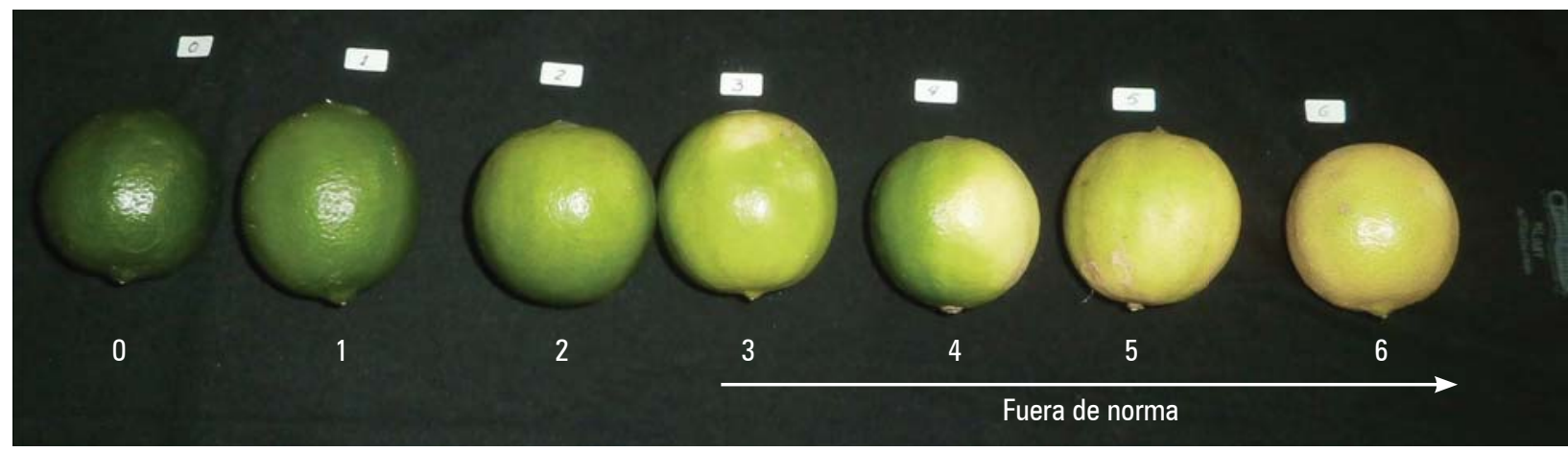

Figura 1. Tabla de color de lima Tahití del Tolima. 0: verde oscuro, 1: verde, 2: verde claro, 3: pocos visos amarillos, 4: muchos visos amarillos, 5: amarillo verdoso y 6 amarillo.

Las anteriores variables se midieron al momento de la cosecha (0) y 15, 30, 45 y $60 \mathrm{ddc}$, tanto para los frutos cosechados oportunamente, como para los frutos cosechados 1, 3, 6 y 9 dac y fueron analizados para cada patrón, según la Norma NTC 4087 (Icontec, 1997).

Los frutos se calificaron con el propósito de cuantificar la probabilidad del cumplimiento de la norma para las muestras, para lo cual se seleccionaron seis variables (parámetros de la tabla 2) que hacen parte de la norma de exportación de limas según NTC 4087. Cada fruto fue calificado con los valores numéricos 1 (cumple) y 0 (no cumple), dependiendo de que estuviera dentro de los rangos mínimos de exigencia con todos los parámetros de la Norma.

\section{Análisis estadístico}

El análisis estadístico se realizó mediante el diseño de bloques completos al azar en cuatro replicaciones usando ANOVA de una vía seguido por una separación de medias usando LSD (Least Square Difference) $(\alpha=0,05)$. Los análisis se realizaron para todas las variables descritas independientemente para comparar los diferentes tiempos en ddc o dac por cada uno de los patrones. Adicionalmente, se hizo un análisis para comparar patrones para cada uno de los ddc o dac. Los datos se analizaron en JMP 11.0.0 (SAS Institute, 2013), fueron evaluados por variable y en caso de encontrar valores atípicos, estos fueron eliminados luego de evaluar las razones.
Los datos se tabularon usando la media \pm desviación estándar. Durante el proceso de análisis, los datos fueron transformados usando el logaritmo o la raíz cuadrada dependiendo de las necesidades y las características de las muestras, con el propósito de mantener las suposiciones del análisis de varianza y la separación de las medias.

\section{RESULTADOS Y DISCUSIÓN}

\section{Comportamiento poscosecha en almacenamiento de los patrones evaluados para cosecha oportuna}

\section{Diámetro transversal}

El análisis de la variación del diámetro transversal de los frutos de la lima durante el almacenamiento se realizó mediante regresión, encontrándose una pendiente negativa para todos los patrones. Es decir, la relación es inversa, indicando que a medida que transcurre el periodo de almacenamiento, disminuye el diámetro transversal de los frutos. El coeficiente de correlación $R^{2}$ osciló entre 0,825 y $0,949(\alpha=0,05)$. Es de destacar que estas variaciones de diámetro transversal no afectan el cumplimiento de la Norma 4087 de los frutos cosechados. Los resultados muestran que los frutos de los patrones presentan diámetros transversal entre 5,56 $00,22 \mathrm{~cm}$ para 'Carrizo' y 4,68 $\pm 0,22$ $\mathrm{cm}$ para 'S $\mathrm{SE}$ ', después de $60 \mathrm{~d}$ de almacenamiento, los cuales cumplen con la exigencia de la norma $(>4,2$

Tabla 1. Firmeza para frutos de lima ácida Tahití según NTC 4087 (Icontec, 1997).

\begin{tabular}{|c|c|c|c|c|c|}
\hline Firmeza & $<0,1471 \mathrm{kPa}$ & $0,1472-0,1969 \mathrm{kPa}$ & $0,1970-0,2215 \mathrm{kPa}$ & $0,2216-0,2459 \mathrm{kPa}$ & $>0,2460 \mathrm{kPa}$ \\
\hline \multirow{2}{*}{ Escala } & 0 & 1 & 2 & 3 & 4 \\
\hline & En norma & & Fuera de norma & & \\
\hline
\end{tabular}


$\mathrm{cm})$. Resultados similares fueron encontrados por Vanegas (2002) y Bassan et al. (2016) para frutos de lima ácida Tahití.

\section{Peso fresco del fruto}

El análisis de la variación de peso fresco de los frutos de lima durante el almacenamiento se realizó mediante regresión, encontrándose una pendiente negativa para todos los patrones, con coeficiente de determinación $R^{2}$ de $0,84(\alpha=0,05)$. Es decir, a medida que transcurre el tiempo, los frutos pierden peso. Estas variaciones de peso no afectan el cumplimiento de la norma de los frutos cosechados. Los resultados presentan un comportamiento similar a los obtenidos en el Salvador por Vanegas (2002), donde se reporta una pérdida de peso del 1\% durante los primeros $10 \mathrm{~d}$ y $0,3 \%$ en los siguientes $5 \mathrm{~d}$ y en Brasil por Silva et al. (2016).

El peso fresco de los frutos de los patrones varía entre un máximo de 103,91 $\pm 6,43$ g para el patrón Carrizo y un mínimo de 64,28 $\pm 4,95 \mathrm{~g}$ para el 'Volkameriano', lo que indica que todos los patrones cumplen plenamente con la norma (> $42 \mathrm{~g}$ ) hasta los $60 \mathrm{~d}$ después de almacenados los frutos. Estos resultados muestran que el peso de los frutos hasta $30 \mathrm{ddc}$ es superior al reportado por otros países exportadores como Brasil (Silva et al., 2016) y el Salvador que registra pesos de 78,8 g (Vanegas, 2002).

\section{Color de los frutos}

De acuerdo con la escala dada en la figura 1, los resultados muestran que los frutos hasta los $45 \mathrm{ddc}$ conservan su coloración máxima (2). La excepción es el patrón Volkameriano, que en promedio está por encima de la norma y sin embargo, no es estadísticamente diferente $(P>0,05)$ de los otros patrones. A los $60 \mathrm{ddc}$ no hubo diferencias entre los patrones, estando todos ellos fuera de norma. Trabajos realizados por la Federación Nacional de Cafeteros de Colombia (Chaparro et al., 2004) muestran que las limas presentan una coloración verde oscura brillante, óptima para exportación, lo cual concuerda con los resultados encontrados en la presente investigación hasta los $45 \mathrm{ddc}$.

\section{Porcentaje del contenido de jugo con relación al peso fruto}

Los resultados obtenidos muestran que el peso del jugo se encuentra entre máximo 47,15 $\pm 5,33 \mathrm{~g}$
(46,22\% en peso) para el patrón SxE y mínimo $21,48 \pm 1,92$ g (32,6\% en peso) para el patrón Kryder, los cuales cumplen con la norma hasta los $30 \mathrm{ddc}$, la cual establece un valor mínimo de $27 \%$ de contenido de jugo. Los análisis estadísticos realizados a los 0 , $15,30,45 \mathrm{ddc}$ muestran que no hay diferencias entre los diferentes patrones $(P>0,05)$ para esta variable. A los 45 y $60 \mathrm{ddc}$ los patrones se caracterizaron por un bajo contenido de jugo estando por encima del límite de $27 \%$. Resultados similares a los obtenidos fueron registrados por Corpoica (Yadira, 2011) con valores medios de $32 \%$ del peso del jugo.

\section{Acidez total titulable}

Los resultados muestran que el porcentaje de ácido cítrico varió entre 4,90 $\pm 0,32 \%$ para frutos del patrón Carrizo y $3,05 \pm 0,09 \%$ para frutos del patrón Cleopatra, indicando que los frutos de todos los patrones cumplen con la norma (inferior al 5\% de ácido cítrico). El análisis LSD muestra que no hay diferencias estadísticas entre los patrones hasta $15 \mathrm{ddc}$, las cuales se empiezan a notar a partir de $30 \mathrm{ddc}(P \leq 0,05)$. Resultados similares fueron encontrados por Chaparro et al. (2004) con contenido de ácido cítrico del 5\% a partir del color 0 .

\section{Firmeza del fruto}

Los resultados obtenidos indican que a partir de los $30 \mathrm{ddc}$ los limones seleccionados no cumplen con la Norma 4087 a excepción de 'Kryder' que se mantiene igual o menor a lo establecido en la escala de la tabla de firmeza (Tab. 1). A los 45 y 60 ddc todos los patrones están fuera de norma y no presentan diferencias estadísticas $(P>0,05)$ entre los patrones. Resultados similares a los encontrados sobre firmeza de los frutos de lima Tahití, registran dos comportamientos diferentes durante el almacenamiento, a saber: se endurecen al suspenderse el proceso de maduración de los frutos por deshidratación de la cáscara (por ser fruto no-climatérico no hay producción autocatalítica de etileno) o se ablandan hasta descomponerse cuando continua el proceso de maduración, si los frutos se protegen de la desecación (Berdeja-Arbeu et al., 2016).

\section{Calificación según la Norma 4087}

Los frutos de los patrones evaluados cumplen con los requisitos de la norma de exportación hasta los 
$15 \mathrm{ddc}$, a partir de este tiempo y hasta los $30 \mathrm{ddc}$ la calificación es para 'Kryder' de 1,00 00,0, 'Carrizo' de

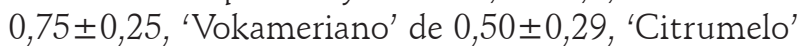
de 0,25 $\pm 0,25$ y 'Cleopatra' de 0,25 $\pm 0,25$. Los patrones tienen una alta probabilidad de cumplir con la norma (únicamente 'Kryder' no cumple) de acuerdo con la consideración expresada en la metodología de 1: si cumple y 0: no cumple (Tab. 2). Después de los $45 \mathrm{ddc}$ ninguno de los patrones seleccionados cumple con todos los requisitos de la norma (Tab. 3).

\section{Evolución de las variables evaluadas para cada uno de los patrones, durante el periodo de almacenamiento de frutos cosechados oportunamente}

\section{Patrón Carrizo}

Los frutos del patrón Carrizo se mantienen hasta los 60 ddc dentro de los parámetros de calidad extra como calibre $\mathrm{C} / \mathrm{D}$, de acuerdo con el peso (entre $67,45 \pm 3,09$ y $103,91 \pm 6,43$ g) y el diámetro (entre $5,02 \pm 0,10$ y $5,56 \pm 0,22 \mathrm{~cm}$ ), según la norma NTC 4087. Los resultados muestran que las variables: diámetro transversal, peso del fruto, color, jugo, firmeza y calificación, presentan diferencias estadísticas en las lecturas hasta los $60 \mathrm{ddc}$ con probabilidades entre $P=0,0001$ y 0,0780 . El mejor comportamiento de las variables se presentó hasta los 30 ddc así: diámetro transversal medio entre 5,56 y $5,19 \mathrm{~cm}$, peso del fruto entre 103,00 y 88,93 g, color $\leq 1$, peso medio del jugo entre 44,90 y 34,20 g, escala de firmeza entre 0 y 1 y calificación de aprobación según la norma. Con el paso del tiempo de almacenamiento la variable que menos cambia es el porcentaje de ácido cítrico y la más crítica es la firmeza (Fig. 2), lo cual se debe al tipo de portainjerto que tienen las limas del estudio (Colauto et al., 2004)

\section{Patrón Citrumelo}

Los frutos se mantienen por tamaño y peso dentro de los parámetros de calidad extra de la norma como calibre C (peso entre 72,13 $\pm 4,75$ y 101,68 $\pm 5,54 \mathrm{~g}$ y diámetro entre 4,88 $\pm 0,10$ y $5,34 \pm 0,05 \mathrm{~cm}$ ) hasta los $45 \mathrm{ddc}$ y como calibre D hasta los $60 \mathrm{ddc}$. Las variables peso del jugo y porcentaje de ácido cítrico no cambian significativamente con el paso del tiempo de almacenamiento. Sin embargo, la calificación muestra que incumple con la norma a partir de los $15 \mathrm{ddc}$

Tabla 2. Parámetros evaluados para determinar la probabilidad de los frutos de cumplir la norma NTC 4087 (Icontec, 1997).

\begin{tabular}{|c|c|c|c|c|c|}
\hline $\begin{array}{c}\text { Diámetro } \\
\text { transversal }\end{array}$ & Peso fresco & $\begin{array}{c}\text { Color } \\
\text { (figura 2-3) }\end{array}$ & $\begin{array}{c}\text { Jugo } \\
(\%)\end{array}$ & $\begin{array}{c}\text { Ácido cítrico } \\
(\%)\end{array}$ & $\begin{array}{c}\text { Escala de firmeza } \\
\text { (tabla 2-1) }\end{array}$ \\
\hline$>4,2 \mathrm{~cm}$ & $>42 \mathrm{~g}$ & $<3$ & $>27 \%$ & $<5 \%$ & $\leq 1$ \\
\hline
\end{tabular}

Tabla 3. Valores medios de escala de calificación según Norma Icontec 4087 de frutos de lima ácida Tahití durante el almacenamiento. Frutos cosechados oportunamente ( \pm desviación estándar).

\begin{tabular}{|l|c|c|c|c|c|}
\hline \multicolumn{7}{|c|}{ Calificación Norma NTC 4087. Media \pm desviación estándar. } \\
\hline \multicolumn{1}{|c|}{ Patrón } & $0 \mathrm{ddc}$ & $15 \mathrm{ddc}$ & $30 \mathrm{ddc}$ & $45 \mathrm{ddc}$ & $60 \mathrm{ddc}$ \\
\hline Carrizo & $0,75 \pm 0,25 \mathrm{a}$ & $0,75 \pm 0,25 \mathrm{a}$ & $0,75 \pm 0,25 \mathrm{ab}$ & $0,0 \pm 0,0 \mathrm{a}$ & $0,0 \pm 0,0 \mathrm{a}$ \\
\hline Citrumelo & $1,00 \pm 0,00 \mathrm{a}$ & $0,75 \pm 0,25 \mathrm{a}$ & $0,25 \pm 0,25 \mathrm{bc}$ & $0,0 \pm 0,0 \mathrm{a}$ & $0,0 \pm 0,0 \mathrm{a}$ \\
\hline Cleopatra & $0,75 \pm 0,25 \mathrm{a}$ & $0,50 \pm 0,29 \mathrm{a}$ & $0,25 \pm 0,25 \mathrm{bc}$ & $0,0 \pm 0,0 \mathrm{a}$ & $0,0 \pm 0,0 \mathrm{a}$ \\
\hline Kryder & $0,75 \pm 0,25 \mathrm{a}$ & $1,00 \pm 0,00 \mathrm{a}$ & $1,00 \pm 0,00 \mathrm{a}$ & $0,0 \pm 0,0 \mathrm{a}$ & $0,0 \pm 0,0 \mathrm{a}$ \\
\hline S $\times$ E & $1,00 \pm 0,00 \mathrm{a}$ & $0,75 \pm 0,25 \mathrm{a}$ & $0,00 \pm 0,00 \mathrm{c}$ & $0,0 \pm 0,0 \mathrm{a}$ & $0,0 \pm 0,0 \mathrm{a}$ \\
\hline Volkameriano & $1,00 \pm 0,00 \mathrm{a}$ & $1,00 \pm 0,00 \mathrm{a}$ & $0,50 \pm 0,29 \mathrm{abc}$ & $0,0 \pm 0,0 \mathrm{a}$ & $0,0 \pm 0,0 \mathrm{a}$ \\
\hline Estadísticas & $\begin{array}{c}\mathrm{t}=2,1314 ; \mathrm{df}=8-23 ; \\
P=0,8779)\end{array}$ & $\begin{array}{c}\mathrm{t}=2,1314 ; \mathrm{df}=8-23 ; \\
P=0,8379)\end{array}$ & $\begin{array}{c}\mathrm{t}=2,1314 ; \mathrm{df}=8-23 ; \\
P=0,1789)\end{array}$ & $\begin{array}{c}(\mathrm{t}=2,1314 ; \mathrm{df}=8-23 ; \\
P=0,00)\end{array}$ & $\begin{array}{c}(\mathrm{t}=2,1314 ; \mathrm{df}=8-23 ; \\
P=0,000)\end{array}$ \\
\hline
\end{tabular}

Promedios con letras distintas indican diferencia significativa según la prueba de $\operatorname{LSD}(P \leq 0,05)$.

Nota: hay una alta probabilidad de cumplir con la norma (1: si cumple y 0: no cumple), los demás patrones no la cumplen. 
$(0,75 \pm 0,25)$ por endurecimiento de la cáscara y pérdida de brillo (Fig. 3), siendo el color verde opaco el que predomina, de acuerdo con la comparación realizada con la carta colorimétrica (Fig. 1).

\section{Patrón Cleopatra}

Los frutos del patrón Cleopatra se mantienen dentro de los parámetros de calidad extra por peso y tamaño con calibre C (peso entre 76,63 $\pm 7,02$ y $97,67 \pm 5,23 \mathrm{~g}$ y diámetro entre 4,95 $\pm 0,16$ y $5,30 \pm 0,07 \mathrm{~cm}$ ) hasta los 60 ddc. En los frutos de este patrón la firmeza es la característica limitante incumpliendo la norma a partir de los $30 \mathrm{ddc}(2,0 \pm 0,41)$. A pesar de una reducción significativa en la calidad, el diámetro, el peso del fruto, el peso del jugo y el porcentaje de ácido cítrico, se mantienen por encima de la norma hasta los 60 ddc. El color se sale de la norma después de los 45 ddc (Fig. 4).

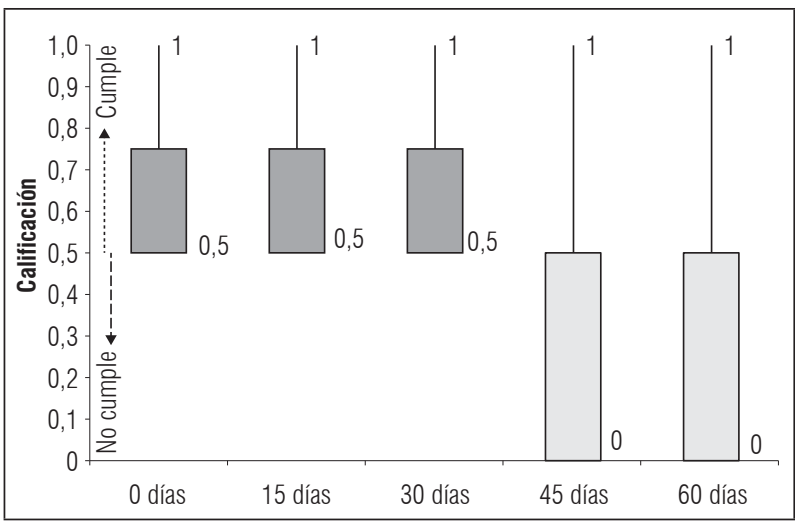

Figura 2. Calificación de los frutos según la NTC 4087 para el patrón Carrizo.

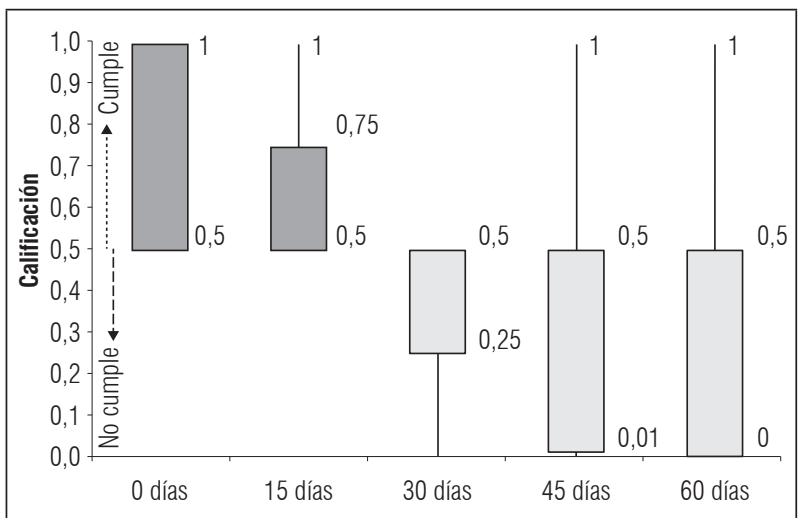

Figura 3. Calificación de los frutos según la NTC 4087 para el patrón Citrumelo.

\section{Patrón Kryder}

Los frutos del patrón Kryder se mantienen dentro de los parámetros de calidad extra por peso y tamaño con calibre $C$ (peso entre $81,08 \pm 4,14$ y $89,73 \pm 4,56$ g y diámetro entre $5,08 \pm 0,08$ y $5,26 \pm 0,08 \mathrm{~cm}$ ) hasta los $60 \mathrm{ddc}$. Los resultados muestran que en estos frutos el porcentaje de ácido cítrico no cambia significativamente en los primeros $60 \mathrm{ddc}$, mientras que el diámetro transversal, peso del fruto, color, porcentaje de jugo, firmeza y calificación cambian significativamente con el tiempo. Sin embargo, las variables diámetro transversal y peso del fruto se mantienen por encima de la norma hasta los $60 \mathrm{ddc}$ (Fig. 5).

\section{Patrón $S \times E$}

Los frutos del patrón $\mathrm{S} \times \mathrm{E}$ se mantienen dentro de los parámetros de calidad extra por peso y tamaño con

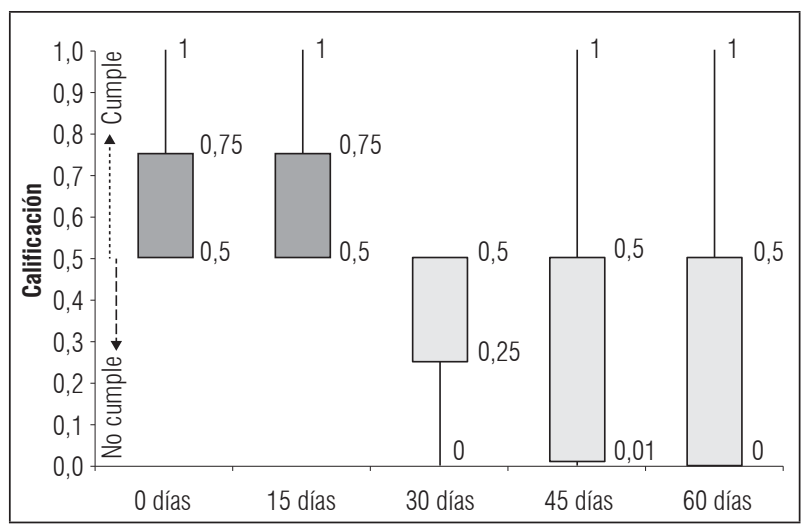

Figura 4. Calificación de los frutos según la NTC 4087 para el patrón Cleopatra.

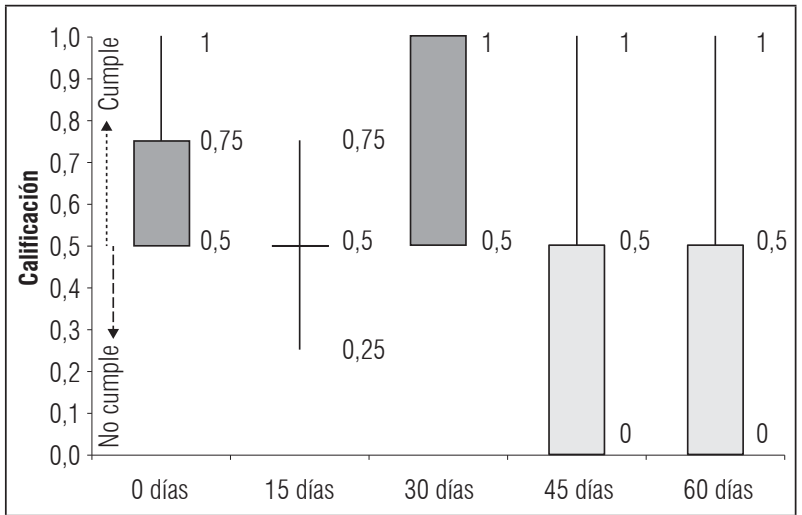

Figura 5. Calificación de los frutos según la NTC 4087 para el patrón Kryder. 
calibre C/D (peso entre 77,60 $\pm 6,40$ y 102,05 $\pm 6,52 \mathrm{~g}$ y diámetro entre $5,03 \pm 0,15$ y $5,53 \pm 0,08 \mathrm{~cm}$ ) hasta los $60 \mathrm{ddc}$. Los resultados muestran que el diámetro transversal, peso del fruto, color, porcentaje de jugo, firmeza y calificación según la Norma NTC 4087, presentan diferencias estadísticas en las lecturas entre 0 y 60 ddc, según pruebas LSD. El porcentaje de ácido cítrico en las lecturas entre 0 y 60 ddc no presenta diferencias (Fig. 6).

\section{Patrón Volkameriano}

Los frutos del patrón Volkameriano se mantienen dentro de los parámetros de calidad extra por peso y tamaño con calibre $\mathrm{C} / \mathrm{D}$ (peso fresco entre $64,28 \pm 4,95$ y $98,63 \pm 10,85$ g y diámetro entre $4,78 \pm 0,13$ y $5,37 \pm 0,20 \mathrm{~cm}$ ) hasta los $60 \mathrm{ddc}$, de acuerdo con la tabla 2. De las variables evaluadas la más crítica

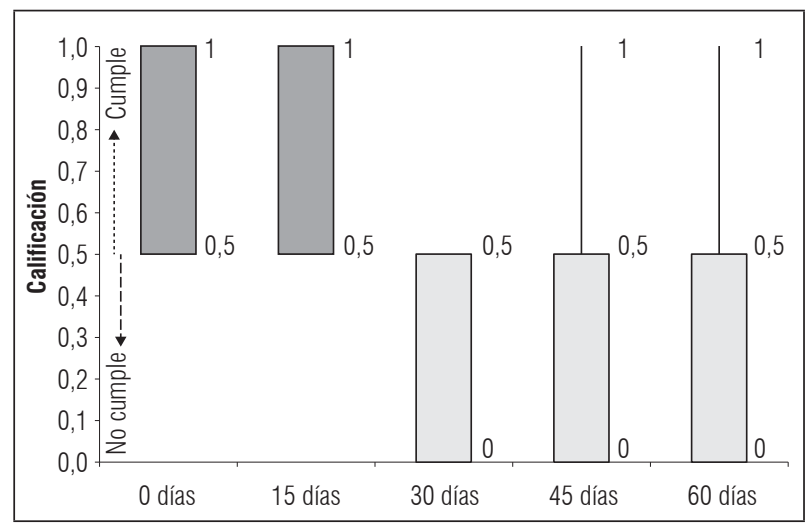

Figura 6. Calificación de los frutos según la NTC 4087 para el patrón $\mathrm{S} \times \mathrm{E}$.

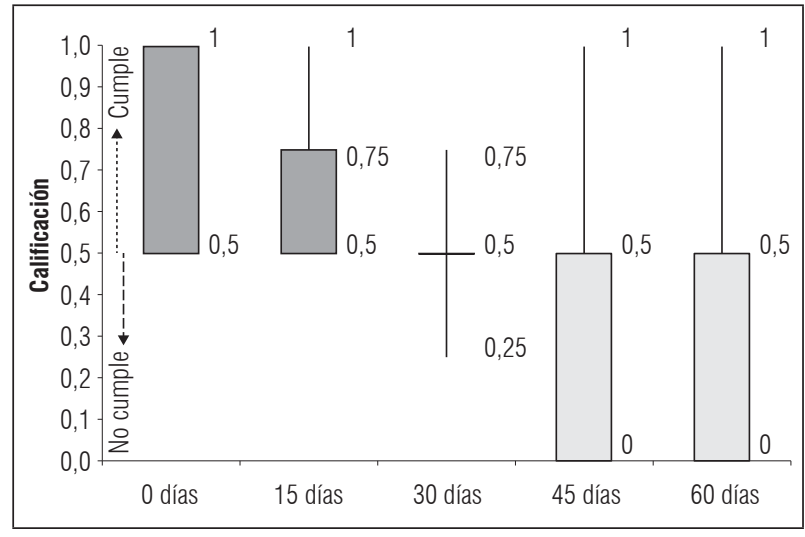

Figura 7. Calificación de los frutos según la NTC 4087 para el patrón Volkameriano. es la firmeza, que se sale de la norma a los 30 ddc $(2,25 \pm 0,25)$. El mejor comportamiento de las variables evaluadas se presentó hasta los 30 ddc así: peso fresco de fruto (entre 98,63 y 78,83 g), color $(<2)$ y calificación de aprobación según la norma. El peso del jugo en las lecturas entre 0 y $60 \mathrm{ddc}$ no presenta diferencias estadísticas (Fig. 7).

\section{Influencia del momento de recolección, en las características de calidad poscosecha de frutos de seis patrones de lima ácida Tahití}

Los resultados del segundo muestreo indican que realizar la cosecha 1, 3, 6 y $9 \mathrm{~d}$ antes del momento de la cosecha comercial no tiene efecto adicional sobre la calidad de los frutos y no prolonga el tiempo de almacenamiento.

\section{Calificación}

Los resultados de la lectura de la calificación de los frutos cosechados 1, 3, 6 y 9 dac muestran que estos frutos cumplen con la norma en todas las variables hasta los $15 \mathrm{ddc}$. A partir de los $30 \mathrm{ddc}$ los frutos de todos los patrones incumplen con la norma, indicando que cosechar los frutos antes de tiempo no aumenta la vida útil de almacenamiento (Tab. 4).

\section{Sólidos solubles totales}

El contenido de SST en los patrones para los frutos cosechados oportunamente y los cosechados 1, 3, 6 , y 9 dac se incrementan $\pm 4^{\circ}$ Brix durante todo el almacenamiento, debido, supuestamente, a la concentración de azúcares en el jugo, por degradación de los polisacáridos en la membrana celular (Yahia et al., 2011). Los valores varían entre 8 y $11^{\circ} \mathrm{Brix}$ para todos los patrones. Resultados similares fueron encontrados en El Salvador (Vanegas, 2002).

\section{Grosor de la cáscara}

Se observa en todos los patrones que el grosor de la cáscara de los frutos cosechados oportunamente y los cosechados 1, 3, 6 y $9 \mathrm{ddc}$, disminuye durante el almacenamiento, variando de $2,0 \mathrm{~mm}$ al inicio del almacenamiento hasta $0,4 \mathrm{~mm}$ al final. Resultados similares fueron encontrados en El Salvador por Vanegas (2002). 
Tabla 4. Valores medios de escala de calificación (de acuerdo con la NTC 4087) de frutos de seis patrones de lima ácida Tahití durante la poscosecha, recolectados 1, 3, 6 y 9 DAC.

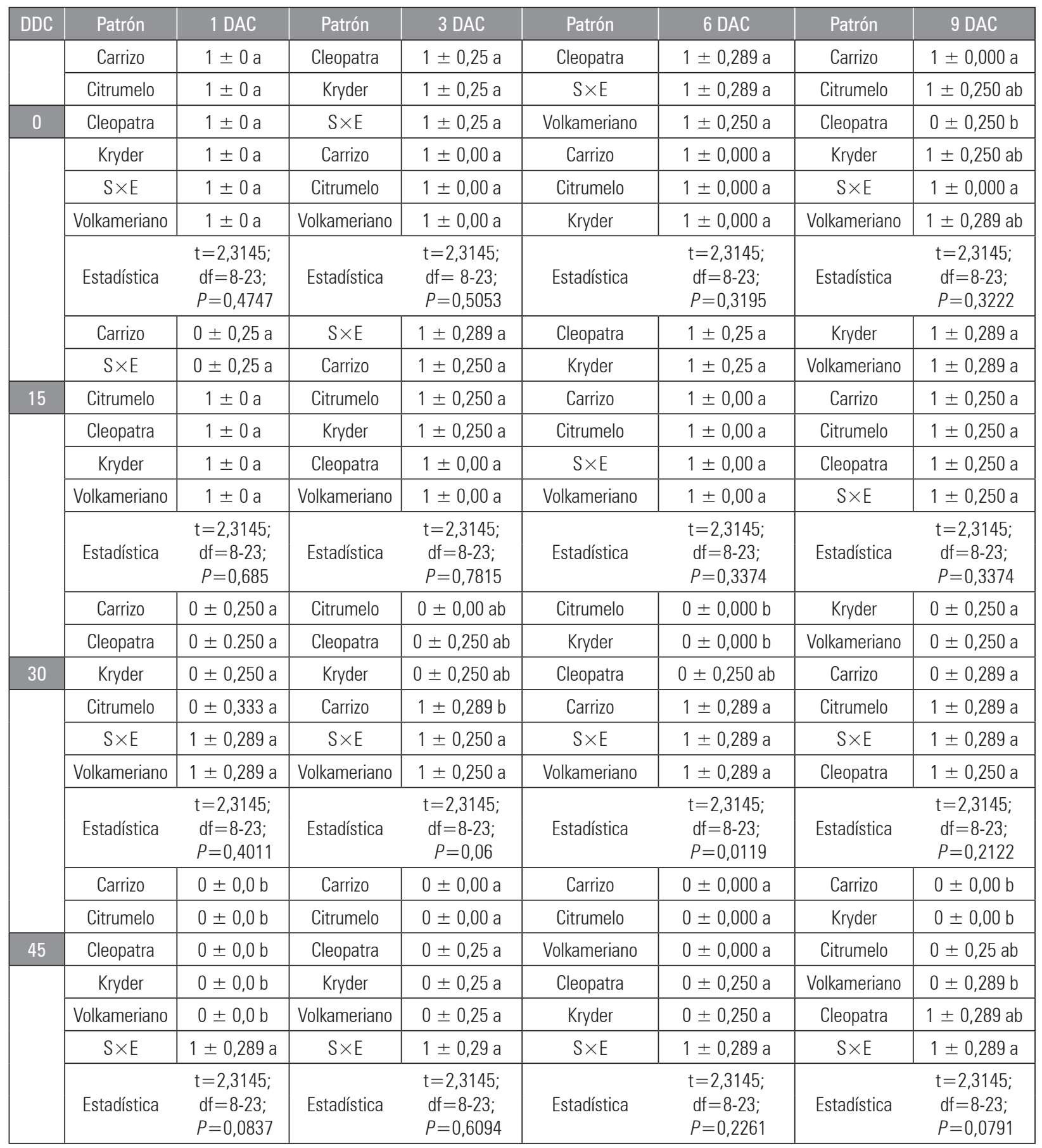

Nota: hay una alta probabilidad de cumplir con la Norma (1: si cumple y 0: no cumple), los demás patrones no la cumplen.

\section{Daños fitosanitarios en poscosecha}

Para observar los daños fitosanitarios causados por factores bióticos o las fisiopatías, se colectaron 400 frutos y se observaron semanalmente durante el ensayo hasta los $60 \mathrm{ddc}$. Los resultados mostraron que 48 frutos (12\%) fueron rechazados durante todo el ensayo y de estos el 79,2\% (38 frutos) presentaron 
ataque del hongo Phytophthora ssp. Los otros 10 frutos presentaron daños por moho verde, Penicillium digitatum y moho azul Penicillium italicum, raspaduras por ácaros y Trips.

\section{CONCLUSIONES}

- Los frutos de lima ácida Tahití, cosechados en el momento oportuno en el C.I. Nataima, sobre los patrones Sunki $\times$ English, Carrizo, Volkameriano, Cleopatra, Citrumelo, Kryder, y almacenados durante $60 \mathrm{ddc}$, conservaron sus características de calibre (diámetro y peso fresco), peso del jugo y porcentaje de ácido cítrico durante este tiempo. Las características de firmeza y color para exportación se mantienen entre los 15 y $30 \mathrm{ddc}$ para frutos sobre estos patrones. 'Kryder' y 'Carrizo' son los únicos patrones que conservan las características de exportación de la lima Tahití entre los 30 y 45 ddc.

- Las variables físicas de color y firmeza son las que presentan más cambios durante el almacenamiento, antes de 30 ddc. El color de las limas cambia de verde oscuro brillante característico a un verde claro $\mathrm{y}$ aparecen visos amarillos hasta que los frutos se vuelven completamente amarillos. Con respecto a la firmeza, cuando la cáscara pierde agua los frutos se endurecen durante el almacenamiento, ofreciendo una resistencia superior a $0,1970 \mathrm{kPa}$, lo cual hace que los frutos no sean aptos para exportación.

- El diámetro transversal, peso del fruto, peso del jugo, porcentaje de ácido cítrico y contenido de SST se conservan hasta los $45 \mathrm{ddc}$ y cumplen con la norma de exportación.

- Cosechar los frutos antes de su madurez comercial no permitió prolongar el tiempo de almacenamiento.

- Los frutos presentaron bajos porcentajes de defectos durante el almacenamiento, causados principalmente por Phytophthora ssp. y Oleocelosis. Se presentaron pocos daños causados por raspaduras, ácaros y trips, moho verde (Penicillium digitatum) y moho azul (Penicillium italicum).

\section{AGRADECIMIENTOS}

Los autores agradecen a la Corporación Colombiana de Investigación Agropecuaria (Corpoica) y a la Universidad Nacional de Colombia, sede Bogotá, por su apoyo a esta investigación.

\section{REFERENCIAS BIBLIOGRÁFICAS}

Bassan, M.M., F.A.A. Mourão Filho, R.F. Alves, D.F. Bezerra, H.T.Z. Couto y A.P. Jacomino. 2016. Beneficiamento pós-colheita de lima ácida 'Tahiti' afeta sua qualidade e conservação. Ciênc. Rural 46(1), 184-190. Doi: 10.1590/0103-8478cr20141492.

Berdeja-Arbeu, R., L. Aguilar-Méndez, D. Moreno-Velázquez, G. Vázquez-Huerta, A. Ibáñez-Martínez y R. Ontiveros.Caburata. 2016. Calidad de fruta de lima 'Persa' en diferentes portainjertos en Veracruz, México. Acta Agríc. Pecu. 2(1), 17-22.

Caicedo, A. 2000. Necesidades hídricas de la lima ácida Tahití. Corporación Colombiana de Investigación Agropecuaria - Corpoica, C.I. Nataima, El Espinal, Colombia.

Cañizares C., A., O. Bonafine, J.R. Méndez N., D. Laverde y R. Puesme. 2012. Crecimiento, desarrollo y calidad de frutos de Lima 'Tahitî' (Citrus latifolia Tanaka) en el Estado Monagas, Venezuela. Rev. Cient. UDO Agríc. $12(3), 535-541$.

Chaparro, M.C., A. Rojas, M. Peñuela, P. Gómez, V. Aristizábal y R. López. 2004. Caracterización de productos hortofrutícolas colombianos y establecimiento de las normas de calidad. Cenicafé, Chinchiná, Colombia.

Coelho, F., M. Coelho y A. Magalhães. 2004. Irrigação e fertirrigação em citros. Circular Técnica 72. Embrapa, Cruz das Almas, Brasil.

Colauto, S.N.M. y J.V. Vieira. 2004 Porta-enxertos para a limeira ácida 'Tahiti'. Sci. Agric. 61(2). Doi: 10.1590/ S0103-9016200400020000

Dorado, D.Y. 2011. Influencia del riego y la fertilización en el rendimiento y calidad de lima ácida Tahití (Citrus latifolia Tanaka). Tesis de maestría. Facultad de Ciencias Agropecuarias, Universidad Nacional de Colombia, Palmira, Colombia

Dorado, D., L.C. Grajales y L. Ríos. 2015. Efecto del riego y la fertilización sobre el rendimiento y la calidad de la fruta de lima ácida Tahití Citrus latifolia Tanaka (Rutaceae). Cienc. Tecnol. Agropecu. 16(1), 87-93.

Ebratt, E.E., L.T. Rubio, V.A. Costa, E.M. Zambrano, A.P. Castro y M.Y. Santamaría. 2011a. Primer registro de Tamarixia radiata (Waterston, 1922) (Hymenoptera: Eulophidae) en Colombia. Rev. Fac. Nal. Agr. Mede1lín. 64(2), 6141-6146.

Ebratt, E.E., L.T. Rubio, V.A. Costa, A.P. Castro, E.M. Zambrano y J. Ángel. 2011b. Diaphorina citri (Kuwayama, 1907) and Tamarixia radiata (Waterston, 1922) in citrus crops of Cundinamarca, Colombia. Agron. Colomb. 29(3), 487-493.

Hernández, D.R., D. Mateus y J.O. Orduz-Rodríguez. 2014. Características climáticas y balance hídrico de la lima ácida Tahití (Citrus latifolia Tanaka) en cinco localidades productoras de Colombia. Rev. Colomb. Cienc. Hortic. 8(2), 217-229. 
Icontec. 1997. Norma Técnica Colombiana NTC 4087. Frutas frescas. Lima Tahití. Especificaciones. Instituto Colombiano de Normas Técnicas y Certificación, Bogotá.

IGAC. 2016. Mapa de la subdirección de Agroecología. En: Mapas de Colombia, http://geoportal.igac.gov. co:8888/siga_sig/Agrologia.seam; consulta: 3 de septiembre de 2016

Orduz-Rodríguez, J.O. 2012. Cítricos (Citrus spp.). pp. 393420. En Fischer, G. (ed.). Manual para el cultivo de frutales en el trópico. Produmedios, Bogotá.

Rodríguez, M. 2002. Guía técnica. Cultivo de limón pérsico. Centro Nacional de Tecnología Agropecuaria y Forestal - CENTA, San Salvador, El Salvador.

Santos, C.Q. de J., E.A. Girard, V.E. Lima, C.A. da S. Ledo y W. dos S.S. Filho. 2015. Tamanho ótimo de amostras de frutos e de sementes para determinação da poliembrionia em citros. Rev. Bras. Frutic. 37 (1), 172-178. Doi: 10.1590/0100-2945-063/14
Soares, L.A. dos A., M. E. B. Brito, P.D. Fernandes, G.S. de Lima, F. Soares, dos S. Walter y E. S. de O. Filho. 2015. Crescimento de combinações copa - porta-enxerto de citros sob estresse hídrico em casa de vegetação. Rev. Bras. Eng. Agríc. Ambiental, 19(3), 211-217. Doi: 10.1590/1807-1929/agriambi.v19n3p211-217

Silva, J.T.A., I.P. Silva y F.R. Simão. 2016. Produção e nutrição de limoeiro 'Tahiti' em função da adubação com nitrogênio e potássio em cinco safras. Pesq. Agropecu. Bras. 51 (4), 357-363. Doi: 10.1590/ S0100-204X2016000400008

Vanegas, M.J. 2002. Guía técnica del cultivo del limón pérsico. Ministerio de Agricultura y Ganadería. Programa MAG - Frutales. La Libertad, El Salvador.

Yahia, E.M., J. Ornelas-Paz y A. Elansari, A. 2011. Postharvest technologies to maintain the quality of tropical and subtropical fruits. pp. 142-193. In: Yahia, E.M (ed.). Postharvest biology and technology of tropical and subtropical fruits. Vol. 1. Fundamental issues. Woodhead Publishing Ltd., Cambridge, UK. 